\title{
CT-based classification systems for intra-articular calcaneal fractures: the inter- and intra-observer variations as well as integrality
}

\section{zhongzheng wang}

Hebei Medical University Third Affiliated Hospital

\section{Shaobo Liang}

Hebei Medical University Third Affiliated Hospital

\section{Yuchuan Wang}

Hebei Medical University Third Affiliated Hospital

\section{Ze Gao}

Hebei Medical University Third Affiliated Hospital

\section{Siyu Tian}

Hebei Medical University Third Affiliated Hospital

Kuo Zhao

Hebei Medical University Third Affiliated Hospital

\section{Wei Chen}

Hebei Medical University Third Affiliated Hospital

\section{Zhiyong Hou}

Hebei Medical University Third Affiliated Hospital

Yingze Zhang ( $\nabla$ drzyzhangyingze@163.com )

the Third Hospital of Hebei Medical University

\section{Research article}

Keywords: Calcaneal fractures, Classification, Interobserver reliability, Intraobserver reproducibility, Integrality

Posted Date: December 28th, 2020

DOl: https://doi.org/10.21203/rs.3.rs-132350/v1

License: (c) (1) This work is licensed under a Creative Commons Attribution 4.0 International License.

Read Full License 
Version of Record: A version of this preprint was published at The Journal of Foot and Ankle Surgery on December 1st, 2021. See the published version at https://doi.org/10.1053/j.jfas.2021.12.007. 
1 CT-based classification systems for intra-articular calcaneal fractures:

2 the inter- and intra-observer variations as well as integrality

3 Zhongzheng Wang ${ }^{1,3^{\dagger}}$, Shaobo Liang ${ }^{2 \dagger}$, Yuchuan Wang ${ }^{1,3}, Z^{2} \mathrm{Gao}^{1}$, Siyu Tian ${ }^{1,3}$, Kuo

$4 \quad$ Zhao $^{1,3}$, Wei Chen ${ }^{1,3,4}$, Zhiyong Hou ${ }^{1,3,4}$, Yingze Zhang ${ }^{1,3,4^{*}}$

$5{ }^{1}$ Department of Orthopaedic Surgery, the Third Hospital of Hebei Medical University,

6 Tianjin 300100, Shijiazhuang 050051, Hebei, P. R. China.

$7 \quad{ }^{2}$ Department of Pelvic and Acetabular Surgery, Honghui Hospital, Xi' an Jiaotong

8 University, Xi’ an, Shanxi 712000, PR China.

$9{ }^{3}$ Key Laboratory of Biomechanics of Hebei Province, Shijiazhuang 050051, PR

10 China.

$11{ }^{4}$ NHC Key Laboratory of Intelligent Orthopaedic Equipment, Shijiazhuang 050051,

12 PR China.

13 Zhongzheng Wang and Shaobo Liang contributed equally to this work and should be

14 regarded as co-first authors.

15 *Yingze Zhang (Corresponding author)

16 [Affiliation] Department of Orthopedic Surgery, The Third Hospital of Hebei Medical

17 University

18 [Postal address] No. 139 Ziqiang Road, Qiaoxi District, Shijiazhuang, 050051, Hebei

19 Province, China

20 [Phone number] +86 17803210200

21 [Fax number] 0086-0311-87023626

22 [E-mail] drzyzhangyingze@163.com 


\section{$24 \quad$ Abstract}

Background: Several primary fracture classification systems (FCSs) have been widely used for intra-articular calcaneal fractures. The purpose of this study was to measure the inter- and intra-observer variations as well as integrality of the Zwipp, Crosby-Fitzgibbons, Sanders, and Eastwood-Atkins classification systems based on more accurate CT scans.

Methods: 549 patients with intra-articular calcaneal fractures taken from a database in our level-I trauma centre (3 affiliated hospitals) were included from January 2018 to December 2019. For each case, normative CT (1 mm slices) scans were available. Four different observers reviewed all CT scans two times according to these 4 most prevalent FCSs within a 2-month interval. For these four FCSs, the kappa [к] coefficient was used to evaluate interobserver reliability and intraobserver reproducibility, and the percentage that can be classified was used to indicate integrality.

Results: The $\kappa$ values were measured for Zwipp ( $\kappa=0.38$ interobserver, $\kappa=0.61$ intraobserver), Crosby-Fitzgibbons $(\kappa=0.48$ interobserver, $\kappa=0.79$ intraobserver), Sanders $(\kappa=0.40$ interobserver, $\kappa=0.57$ intraobserver $)$, and Eastwood-Atkins $(\kappa=0.44$ interobserver, $\kappa=0.72$ intraobserver). Furthermore, the integrality were calculated for Zwipp (100 \%), Crosby-Fitzgibbons (100\%), Sanders $(92 \%)$ as well as Eastwood-Atkins (89.6\%).

Conclusion: Compared with previous literatures, CT scanning with higher accuracy 
can significantly improve intraobserver reproducibility of Zwipp and Eastwood-Atkins FCSs, but it has no positive effect on variability of Sanders FCS and interobserver reliability of Crosby-Fitzgibbons FCS. While in terms of integrality, Zwipp and Crosby-Fitzgibbons FCSs appear to be superior to the other two FCSs.

Keywords: Calcaneal fractures; Classification; Interobserver reliability; Intraobserver reproducibility; Integrality

\section{Background}

The calcaneus has a complex anatomical structure, is one of the largest and most easily damaged tarsal bone of the foot, and supports the axial load of the body weight [1]. Calcaneal fractures account for approximately $1 \%-4 \%$ of all adult fractures [2-4]. Based on whether the fracture line involves the posterior facet, calcaneal fractures are divided into two categories intra- and extra-articular fractures. Among them, about $70 \%-75 \%$ of calcaneal fractures belong to the former $[5,6]$. However, despite extensive clinical experience of these injuries, the complex and displaced intra-articular calcaneal fractures remain difficult to treat. Over the past few decades, more than a dozen diverse fracture classification systems (FCSs) for intra-articular calcaneal fractures have been proposed but the optimal FCS remains controversial [7]. In the mid-1980s, with the introduction of CT scan imaging technique, CT-based FCSs for intra-articular calcaneal fractures came into being and showed improved correlation with treatment in comparison with the FCSs based on conventional X-ray [7-9]. Several FCSs have been described in previous literatures. While the most 
commonly used FCSs are those of Zwipp [10], Crosby-Fitzgibbons [11], Sanders [12], and Eastwood-Atkins [13], classification systems, and all of them have been confirmed to have a good correlation between fracture types and clinical prognosis $[6,7,14]$. However, some reports suggested that these FCSs have only fair-to-moderate consistency, and no author assessed the integrality of FCSs $[6,7,14,15]$. Therefore, these greatly limited the application of these FCSs in communicating with other orthopaedic surgeons, guiding treatment, and predicting prognosis. In recent years, due to the advancement of thin-layer CT scanning, multi-planar reconstructions (MPR) and volume rendering (VR) reconstruction CT technology, intra-articular fracture line extension and bone fragment displacement can be better visualized and characterized $[2,16]$. Therefore, the authors hypothesized that CT imaging technology with higher accuracy could improve the consistency of FCSs.

The purpose of this study was twofold: 1) to evaluate the interobserver reliability and intraobserver reproducibility of the 4 most prevalent CT-based FCSs; 2) to calculate the integrality of the 4 FCSs and introduce the characteristics of fracture types that cannot be classified.

\section{Methods}

\section{Patients and methods}

This study retrospectively reviewed all consecutive patients with unilateral or bilateral calcaneal fractures who had undergone hospitalization in the same level-I trauma centre (3 affiliated hospitals) from January 2018 to December 2019. The eligibility 
criteria were defined as follows: adult patients (age $\geq 18$ years), diagnosis of closed, unilateral or bilateral intra-articular calcaneal fractures; and standard CT scans from two planes with $1 \mathrm{~mm}$ slices (sagittal, axial and coronal) were available. The exclusion criteria included patients younger than 18 years, patients with open fractures or extra-articular calcaneal fractures, previous calcaneal fracture and previous trauma or surgery involving the calcaneus.

According to the Zwipp [10], Crosby-Fitzgibbons [11], Sanders [12], and Eastwood-Atkins [13], FCSs, four reviewers (2 senior orthopaedic surgeons (A and B) with experience over 10 years; 2 trauma surgical residents $(C$ and $D)$ with experience 6 and 4 years in orthopaedic trauma, respectively) were recruited to classify the images of calcaneal fractures twice, with two months in between. Before reviewing the images, the four reviewers were trained in using the 4 classification systems, provided with the original literature and showed examples of each fracture type. The details of how to analyze the 4 FCSs are shown in Table 1. All personal information in the image data was removed by individuals external to our study. Then, the four reviewers collectively reviewed all the processed image data according to the 4 classification methods. Finally, the four reviewers jointly determined the final fracture types according to each FCS. The classification results were recorded by a person external to the study.

This study was approved by the Ethics Committee of the Third Hospital of Hebei Medical University, according to the Helsinki Declaration, and written informed consent was obtained from all participants. 
111 Table 1. The details of the 4 most widely used FCSs

\begin{tabular}{lll}
\hline Classification (Years) & Reason and Principle & Types \\
\hline Zwipp[10], 1989 & 5 important fracture fragments and 3 primary joints & $1-8$ points \\
Crosby-Fitzgibbons[11], 1990 & Displacement, comminution & I,II,III \\
Sanders[12], 1993 & Displacement, location and number of fracture lines, comminution & I,IIA,IIB,IIC,IIIAB,IIIAC,IIIBC,IV \\
Eastwood-Atkins[13], 1993 & Relationship between lateral wall and lateral bone fragment & I,II,III \\
\hline
\end{tabular}

112

113

114 Statistical analysis

115 As we all know, an ideal FCS should not only be easy to understand, remember,

116 communicate and master by clinicians, but also include the majority of types of

117 fracture, show low variability among users, guide treatment and indicate prognoses

$118[7,17,18]$. The kappa ( $)$ statistics was used to calculate inter- and intra-observer

119 variations. And interobserver reliability was assessed with Fleiss's kappa [19], while

120 intraobserver reproducibility was evaluated by Cohen's kappa [20]. The $\kappa$ was a

121 coefficient of agreement ranging from +1 to $-1 \quad(+1$ : complete agreement; 0 :

122 agreement no better than chance alone; -1 : no agreement). Type of fracture that could

123 not be classified was described during the classification process. And the integrality of

124 FCS was defined as: FCS can cover the percentage of all fractures.

125 All types of fractures and demographic characteristics of patients were tabulated

126 using a Microsoft Excel spreadsheet (Microsoft Corporation, Redmond WA), and the

127 kappa (к) values were analysed using IBM SPSS Statistics for Windows, version 21.0

128 (IBM Corp, Armonk, NY, USA). The level of kappa (к) values was interpreted

129 according to the guidelines of Landis and Koch's (Table 2) [21]. Continuous data are

130 expressed as the means \pm standard deviations, whereas categorical variables are 
presented as frequencies (percentages).

132 Table 2. Landis and Koch interpretation of kappa ( $\kappa$ ) values[21]

\begin{tabular}{|ll|}
\hline kappa (к) value & Agreement \\
\hline$<0.00$ & Poor \\
0.00 to 0.20 & Slight \\
0.21 to 0.40 & Fair \\
0.41 to 0.60 & Moderate \\
0.61 to 0.80 & Substantial \\
0.81 to 1.00 & Excellent \\
\hline
\end{tabular}

\section{Results}

Participants

136 A total of 614 intra-articular calcaneal fractures in 549 patients (men: 510, 92.9\%; women $39,7.1 \%$ ) with a mean age of $42.0 \pm 10.8$ years (range: $18-75$ ) were included in this study, consisting of 484 patients with fractures on the unilateral side, and the other $65(11.8 \%)$ patients with simultaneous bilateral fractures. The most common mechanism of injury were fall from height (68.9\%), traffic injury $(18.4 \%)$, and other injuries $(12.7 \%)$.

\section{Interobserver reliability}

144 In regard to interobserver reliability, orthopaedic surgeons displayed fair-to-moderate agreement for the Zwipp ( $\kappa=0.38)$, Crosby-Fitzgibbons $(\kappa=0.48)$, Sanders $(\kappa=$ $0.40)$, and Eastwood-Atkins $(\kappa=0.44)$ FCSs, which was shown in table 3. The level of agreement was moderate-to-substantial among the 2 senior orthopaedic surgeons 
148 (A and B) for the 4 FCSs, with a $\kappa$ value of 0.57 (0.48 to 0.66). The 2 trauma surgical

149 residents (C and $\mathrm{D})$ showed fair agreement with $\kappa$ values of $0.30(0.23$ to 0.35$)$. The

150 interobserver reliability of senior orthopaedic surgeons was significantly higher than

151 the trauma surgical residents, $\kappa$ values of 0.57 vs 0.30 .

152 Table 3. Interobserver Reliability of the 4 FCSs for intra-articular calcaneal fractures

\begin{tabular}{|c|c|c|c|c|c|c|c|c|}
\hline \multirow{2}{*}{ Observers } & \multicolumn{2}{|c|}{ Zwipp } & \multicolumn{2}{|c|}{ Crosby-Fitzgibbons } & \multicolumn{2}{|c|}{ Sanders } & \multicolumn{2}{|c|}{ Eastwood-Atkins } \\
\hline & k Statistic & Agreement & k Statistic & Agreement & k Statistic & Agreement & k Statistic & Agreement \\
\hline A-B & 0.58 & Moderate & 0.66 & Substantial & 0.55 & Moderate & 0.48 & Moderate \\
\hline A-C & 0.49 & Moderate & 0.57 & Moderate & 0.53 & Moderate & 0.49 & Moderate \\
\hline A-D & 0.30 & Fair & 0.37 & Fair & 0.30 & Fair & 0.50 & Moderate \\
\hline B-C & 0.39 & Fair & 0.49 & Moderate & 0.43 & Moderate & 0.46 & Moderate \\
\hline B-D & 0.29 & Fair & 0.47 & Moderate & 0.32 & Fair & 0.36 & Fair \\
\hline C-D & 0.23 & Fair & 0.33 & Fair & 0.27 & Fair & 0.35 & Fair \\
\hline Mean & 0.38 & Fair & 0.48 & Moderate & 0.40 & Fair & 0.44 & Moderate \\
\hline
\end{tabular}

153 A and B, 2 senior orthopaedic surgeons with experience over 10 years; C and D, 2 trauma

154 surgical residents with experience 6 and 4 years in orthopaedic trauma, respectively

156 Intraobserver reproducibility and integrality

157 In regard to intraobserver reproducibility, orthopaedic surgeons displayed

158 moderate-to-substantial agreement for the Zwipp $(\kappa=0.61)$, Crosby-Fitzgibbons $(\kappa=$

$1590.79)$, Sanders $(\kappa=0.57)$, and Eastwood-Atkins $(\kappa=0.72)$ FCSs, which was shown in

160 table 4. Compared to the trauma surgical residents, the senior orthopaedic surgeons

161 had significantly higher intraobserver reproducibility (substantial vs moderate). In

162 regard to integrality of FCS, Zwipp and Crosby-Fitzgibbons could cover all types of

163 intra-articular calcaneal fractures. However, 49 cases $(8 \%)$ could not be classified by

164 Sanders classification system, and 64 cases (10.4\%) could not be classified by

165 Eastwood-Atkins classification system (Table 4). 

calcaneal fractures

\begin{tabular}{|c|c|c|c|c|c|c|c|c|c|c|c|c|}
\hline \multirow{2}{*}{ Obervers } & \multicolumn{3}{|c|}{ Zwipp } & \multicolumn{3}{|c|}{ Crosby-Fitzgibbons } & \multicolumn{3}{|c|}{ Sanders } & \multicolumn{3}{|c|}{ Eastwood-Atkins } \\
\hline & k Statistic & Agreement & Integrality & k Statistic & Agreement & Integrality & k Statistic & Agreement & Integrality & k Statistic & Agreement & Integrality \\
\hline $\mathbf{A}$ & 0.69 & Substantial & $100 \%$ & 0.85 & Excellent & $100 \%$ & 0.62 & Substantial & $89.3 \%$ & 0.71 & Substantial & $87.5 \%$ \\
\hline B & 0.74 & Substantial & $100 \%$ & 0.82 & Excellent & $100 \%$ & 0.59 & Moderate & $93.7 \%$ & 0.77 & Substantial & $85.9 \%$ \\
\hline C & 0.56 & Moderate & $100 \%$ & 0.69 & Substantial & $100 \%$ & 0.48 & Moderate & $97.5 \%$ & 0.76 & Substantial & $93.1 \%$ \\
\hline D & 0.48 & Moderate & $100 \%$ & 0.80 & Substantial & $100 \%$ & 0.59 & Moderate & $87.4 \%$ & 0.64 & Substantial & $91.9 \%$ \\
\hline Mean & 0.61 & Substantial & $100 \%$ & 0.79 & Substantial & $100 \%$ & 0.57 & Moderate & $92.0 \%$ & 0.72 & Substantial & $89.6 \%$ \\
\hline
\end{tabular}

168 A and B, 2 senior orthopaedic surgeons with experience over 10 years; C and D, 2 trauma

169 surgical residents with experience 6 and 4 years in orthopaedic trauma, respectively

\section{Discussion}

172 In the past few decades, FCSs have been widely accepted in clinical practice. An ideal

173 FCS should be easy to communicate and use, included lower variability, ability to

174 assess the degree of fracture severity, take the whole spectrum of fracture

175 characteristics into consideration, guide treatment, and predict prognoses $[7,14,15,22]$.

176 However, many commonly used FCSs were somewhat disappointing, which lacking

177 these characteristics [23]. To the best of our knowledge, with the improvement of the

178 accuracy of imaging equipment in recent years, there have been no clinical studies

179 measuring the intra- and inter-observer variability as well as integrality of these 4

180 FCSs for fractures of intra-articular calcaneal.

181 In the 1980s, the advent of CT scanning revolutionized the treatment strategy of

182 calcaneal fractures, and better visually defined the subtalar joint involvement $[2,24]$.

183 Which also led to the development of newer FCSs for intra-articular calcaneal

184 fractures. In 1989, Zwipp et al. [10] were the first to use CT imaging to classify

185 calcaneal fractures. They proposed a FCS including two parts (X-fragment, max 5 
points; Y-joint fracture, max 3 points) that allowed to determine the type of calcaneal fractures, its severity, and the prognosis, and the choice of treatment strategy [25]. In 1990, Crosby and Fitzgibbons [11] described a CT-based FCS, and according to the displacement of articular surface, it was divided into three types (I-nondisplaced, II-displaced, III-comminuted). Besides, they were the first to correlated prognosis with the FCS [26]. In 1993, Sanders et al. [12] developed a FCS from an assessment of 120 displaced calcaneal fractures, which was divided into four primary types (I-IV) based on the number and location of fragments of posterior talus face showed on coronal and axial CT images. In the same year, Eastwood and Atkins [13] described a FCS, which was divided into three types (I-III) according to the composition of the fractured lateral wall of the calcaneum. However, despite these FCSs are considered to be relevant to clinical prognosis and have been widely used in clinical practice, their value has been controversial due to their limited reliability and validity.

As previously reported in the literatures, $\kappa$ analysis was widely available in the published studies for Sanders FCS. However, the variability of these data is high $(\kappa$ value range $0.25-0.56$, interobserver; $\kappa$ value range $0.31-0.65$, intraobserver) $[6,14,27]$. The $\kappa$ values for Zwipp FCS in several published studies was 0.24-0.47 (interobserver) and 0.16-0.40 (intraobserver) [6,14]. Schepers et al. [7] reported that the Crosby-Fitzgibbons FCS had moderate interobserver reliability $(\kappa=0.48)$. Besides, Howells et al. [14] indicated that the Eastwood-Atkins FCS had substantial interobserver reliability $(\kappa=0.73)$ and moderate intraobserver reproducibility $(\kappa=$ 0.42). In the present study, our results of Sanders FCS match to the previous 
conclusion. While the results of the other 3 FCSs are obviously different, such as the intraobserver reproducibility of Zwipp and Eastwood-Atkins FCSs are significantly improved, the interobserver reliability of Eastwood-Atkins FCS was lower than the result reported by Howells [14]. Additionally, the results of our study showed that the reliability and reproducibility vary widely among different observers with different training levels and experience. The agreement of senior orthopaedic surgeons (A and B) is significantly better than the trauma surgical residents (C and D). It is contrary to the conclusions of several previous researches [14,28,29].

In regard to integrality of the 4 FCSs, all fracture types can be classified by the FCSs of Zwipp and Crosby-Fitzgibbons. However, 49 cases (8\%) could not be classified by Sanders FCS, and 64 cases (10.4\%) could not be classified by Eastwood-Atkins FCS. The classification of Sanders, based on axial images and the widest undersurface on coronal CT scans, is a commonly used classification for intra-articular calcaneal fractures and uses coronal sections to determine the number and sites of displaced fracture fragments in the posterior facet of the calcaneus [12]. However, the widest undersurface on coronal CT scans is located in the anterior part of the posterior facet, which does not show all parts of posterior facet excellently, and fracture types identified on two adjacent slices of the CT scanning can be inconsistent in the same case [30]. In addition, it seems to be perfect to divide the two fracture lines (line A and B) into medial, central and lateral sides equally, but it is difficult to accurately locate the position of the fracture lines, especially at the junction, in actual classification (Fig. 1). Similar to the Sanders classification, the Eastwood-Atkins 
231 slices of the $\mathrm{CT}$ scanning can be inconsistent in the same case. Furthermore, the

232 Eastwood-Atkins classification only classifies displaced intra-articular calcaneal

233 fractures according to the composition of the fractured lateral wall of the calcaneum,

234 the non-displaced and severe comminuted types cannot be classified (Fig. 2). These

235 may also be one of the main reasons for the high variability of the two FCSs.

236
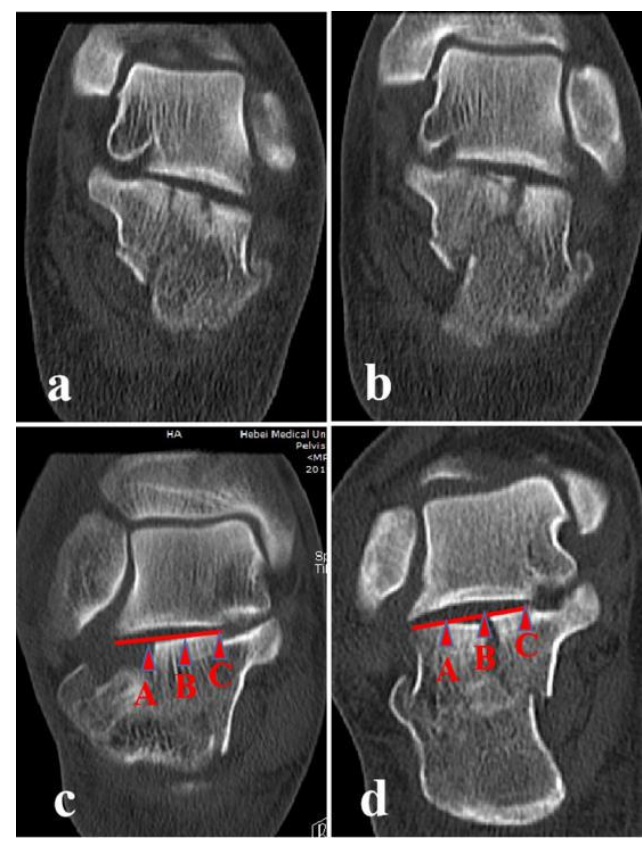

Fig 1. The coronal sections of the widest part of the posterior facet of the calcaneus (a-d) describe three fracture types that cannot be classified or are controversial in the Sanders FCS. The fracture types identified in two adjacent coronal sections of the CT scanning at the widest part are inconsistent in the same case $(\mathbf{a}, \mathbf{b})$. $\mathbf{c}$, the fracture line is located around the junction of line A, and it is difficult to accurately distinguish between type IIA and type IIB. d, the fracture line is located around the junction of line B, and it is difficult to accurately distinguish between type IIB and type IIC.
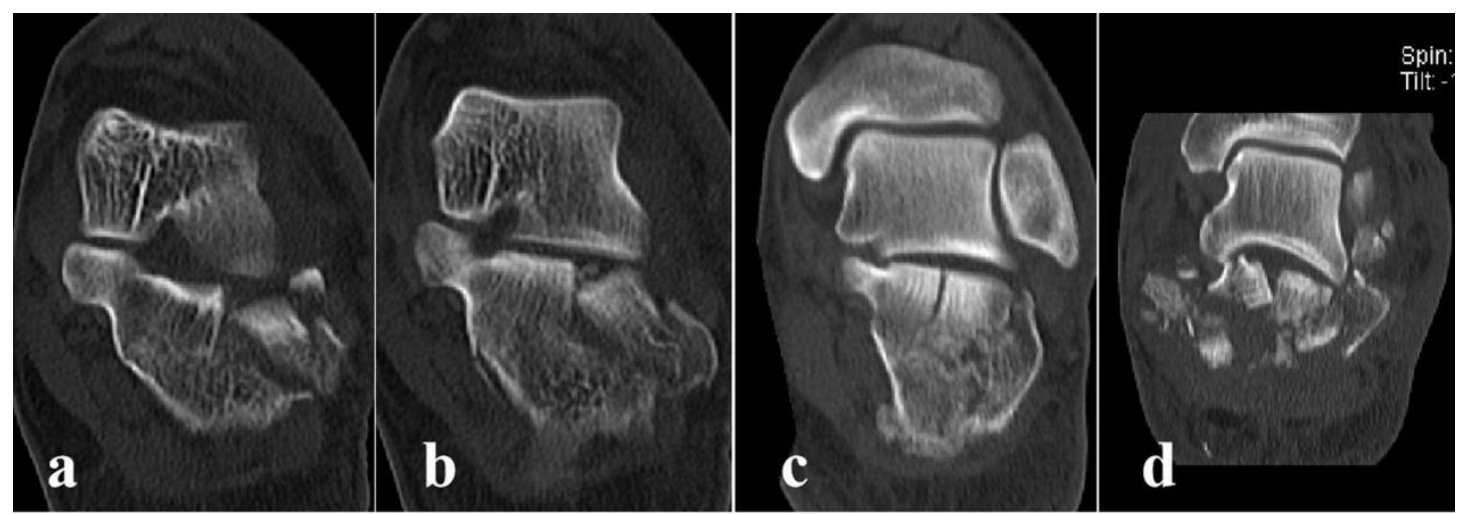
Fig 2. The coronal sections of the posterior facet of the calcaneus (a-d) describe three fracture types that cannot be classified or are controversial in the Eastwood-Atkins FCS. The fracture types identified in two adjacent coronal sections of the CT scan are inconsistent in the same case (a, type III; b, type II). Non-displaced (c) and severe comminuted (d) types are not included in the Eastwood-Atkins FCS.

There are several limitations in our study. One weakness of the present study was the small number of observers used to assess the inter- and intra-observer variations. While, the method that we performed was consistent with many previous studies $[6,14,18,31]$. In addition, the current study was not intended to prove that these 4 FCSs were better or worse than other FCSs. Instead, it was designed to compare the inter- and intra-observer variations as well as integrality of the 4 common FCSs. Furthermore, the Zwipp FCS also includes the extent of soft tissue trauma and other accompanying fractures (max, 4 points). But because this research is a retrospective study, accurate assessment of soft tissue injuries was lacking. Therefore, a prospective study may be more valuable. Finally, this study did not record clinically relevant outcomes and prognostic factors, such as treatment strategy and follow-up, which would be focus of the future research.

\section{Conclusions}

The improvement of the accuracy of CT imaging equipment can be valuable in improving assessment of the intraobserver reproducibility of Zwipp and Eastwood-Atkins FCSs. However, it has no positive effect on the variability of Sanders FCS and the interobserver reliability of Crosby-Fitzgibbons FCS. Furthermore, senior orthopaedic surgeons may have highter agreement than trauma 
surgical residents. Despite the thin-layer CT scanning can more clearly identify

anatomical features of injury, which may have been a contributing factor for improving reproducibility for orthopaedic traumatologists, it cannot compensate for the limitations of the FCS itself. Therefore, surgeons and clinicians should be aware of the limitations regarding the inter- and intra-observer variations as well as integrality for these FCSs, and make targeted improvements to existing FCSs in future researches.

\section{Abbreviations}

FCS: Fracture classification system; CT: Computerized tomography; MPR: multi-planar reconstructions; VR: volume rendering

\section{Acknowledgements}

We appreciate the contribution of all patients, their families, the observers, and the medical staff. We are grateful to all authors.

\section{Authors' contributions}

Yingze Zhang, Zhiyong Hou and Wei Chen conceived the idea for the study; Zhongzheng Wang and Shaobo Liang designed the study. Zhongzheng Wang, Yuchuan Wang, Siyu Tian and Ze Gao of the Department of Orthopedics classified the images of calcaneal fractures. Zhongzheng Wang and Shaobo Liang collected the relevant data; Shaobo Liang and Siyu Tian prepared the figures and tables. Kuo Zhao performed the statistical analyses. All the authors interpreted the data and contributed to preparation of the manuscript. Zhongzheng Wang and Shaobo Liang contributed equally to this manuscript. The authors read and approved the final manuscript.

\section{Funding}

This study was supported by Key project of Hebei Provincial Natural Fund Precision Medicine Joint Fund (No. H2020206456).

\section{Availability of data and materials}

All data used and analyzed during this study are available from the corresponding 
author upon reasonable request.

Ethics approval and consent to participate

This study was approved by the ethics committee of the Third Hospital of Hebei Medical University. Informed consent was obtained from all the participants.

\section{Consent for publication}

Written informed consent was obtained from each patient to authorize the publication of their data.

\section{Competing interests}

The authors declare no conflict of interests regarding the publication of this article.

\section{Author details}

${ }^{1}$ Department of Orthopaedic Surgery, the Third Hospital of Hebei Medical University, Tianjin 300100, Shijiazhuang 050051, Hebei, P. R. China. ${ }^{2}$ Department of Pelvic and Acetabular Surgery, Honghui Hospital, Xi'an Jiaotong University, Xi'an, Shanxi 712000, PR China. ${ }^{3}$ Key Laboratory of Biomechanics of Hebei Province, Shijiazhuang 050051, PR China. ${ }^{4} \mathrm{NHC}$ Key Laboratory of Intelligent Orthopaedic Equipment, Shijiazhuang 050051, PR China.

\section{References}

1. Badillo K, Pacheco JA, Padua SO, Gomez AA, Colon E, Vidal JA. Multidetector CT evaluation of calcaneal fractures. Radiographics. 2011;31(1):81-92. https://doi.org/10.1148/rg.311105036.

2. Galluzzo M, Greco F, Pietragalla M, et al. Calcaneal fractures: radiological and CT evaluation and classification systems. Acta Biomed. 2018;89(1-S):138-150. https://doi.org/10.23750/abm.v89i1-S.7017.

3. Wei N, Zhou Y, Chang W, Zhang Y, Chen W. Displaced Intra-articular Calcaneal Fractures: Classification and Treatment. Orthopedics. 2017;40(6):e921-e929. https://doi.org/10.3928/01477447-20170907-02.

4. Epstein N, Chandran S, Chou L. Current concepts review: intra-articular fractures of the

5. Daftary A, Haims AH, Baumgaertner MR. Fractures of the calcaneus: a review with emphasis

6. Sayed-Noor AS, Agren PH, Wretenberg P. Interobserver reliability and intraobserver reproducibility of three radiological classification systems for intra-articular calcaneal fractures. Foot Ankle Int. 2011;32(9):861-866. https://doi.org/10.3113/FAI.2011.0861. classification: a comparative study. J Foot Ankle Surg. 2009;48(2):156-162. 
ht-tps://doi.org/10.1053/j.jfas.2008.11.006.

8. Schepers T, Ginai AZ, Mulder PG, Patka P. Radiographic evaluation of calcaneal fractures: to measure or not to measure. Skeletal Radiol. 2007;36(9):847-852. https://doi.org/10.1007/s00256-007-0330-6.

9. Guyer BH, Levinsohn EM, Fredrickson BE, Bailey GL, Formikell M. Computed tomography of calcaneal fractures: anatomy, pathology, dosimetry, and clinical relevance. AJR Am J Roentgenol. 1985;145(5):911-919. https://doi.org/10.2214/ajr.145.5.911.

10. Zwipp H, Tscherne H, Wülker N, Grote R. [Intra-articular fracture of the calcaneus. Classification, assessment and surgical procedures]. Unfallchirurg. 1989;92(3):117-129.

11. Crosby LA, Fitzgibbons T. Computerized tomography scanning of acute intra-articular fractures of the calcaneus. A new classification system. J Bone Joint Surg Am. 1990;72(6):852-859.

12. Sanders R, Fortin P, DiPasquale T, Walling A. Operative treatment in 120 displaced intraarticular calcaneal fractures. Results using a prognostic computed tomography scan classification. Clin Orthop Relat Res. 1993(290):87-95.

13. Eastwood DM, Gregg PJ, Atkins RM. Intra-articular fractures of the calcaneum. Part I: Pathological anatomy and classification. J Bone Joint Surg Br. 1993;75(2):183-188. https://doi.org/10.1302/0301-620X.75B2.8444934.

14. Howells NR, Hughes AW, Jackson M, Atkins RM, Livingstone JA. Interobserver and intraobserver reliability assessment of calcaneal fracture classification systems. J Foot Ankle Surg. 2014;53(1):47-51. https://doi.org/10.1053/j.jfas.2013.06.004.

15. Humphrey CA, Dirschl DR, Ellis TJ. Interobserver reliability of a CT-based fracture classification system. J Orthop Trauma. 2005;19(9):616-622. https://doi.org/10.1097/01.bot.0000177107.30837.61.

16. Reginelli A, Zappia M, Barile A, Brunese L. Strategies of imaging after orthopedic surgery. Musculoskelet Surg. 2017;101(Suppl 1):1. https://doi.org/10.1007/s12306-017-0458-z.

17. Vosoughi AR, Shayan Z, Salehi E, Jaberi FM, Solooki S, Kardeh B. Agreement between Sanders classification of intraarticular calcaneal fractures and assessment during the surgery. Foot Ankle Surg. 2020;26(1):94-97. https://doi.org/10.1016/j.fas.2018.12.001.

18. Zhang R, Yin Y, Li A, et al. Three-Column Classification for Acetabular Fractures. The Journal of Bone and Joint Surgery. 2019;101(22):2015-2025. https://doi.org/10.2106/JBJS.19.00284.

19. Senn S. Review of Fleiss, statistical methods for rates and proportions. Res Synth Methods. 2011;2(3):221-222. https://doi.org/10.1002/jrsm.50.

20. Cohen J. Weighted kappa: nominal scale agreement with provision for scaled disagreement or partial credit. Psychol Bull. 1968;70(4):213-220. https://doi.org/10.1037/h0026256.

21. Landis JR, Koch GG. The measurement of observer agreement for categorical data. Biometrics. 1977;33(1):159-174.

22. Bernstein J, Monaghan BA, Silber JS, DeLong WG. Taxonomy and treatment--a classification of fracture classifications. J Bone Joint Surg Br. 1997;79(5):706-707; discussion 708-709. https://doi.org/10.1302/0301-620x.79b5.7177.

23. Bernstein J. Fracture classification systems: do they work and are they useful? J Bone Joint Surg Am. 1994;76(5):792-793.

24. Lauder AJ, Inda DJ, Bott AM, Clare MP, Fitzgibbons TC, Mormino MA. Interobserver and 
intraobserver reliability of two classification systems for intra-articular calcaneal fractures. Foot Ankle Int. 2006;27(4):251-255. https://doi.org/10.1177/107110070602700405.

25. Zwipp H, Tscherne H, Thermann H, Weber T. Osteosynthesis of displaced intraarticular fractures of the calcaneus. Results in 123 cases. Clin Orthop Relat Res. 1993(290):76-86. https://doi.org/10.1097/00003086-199305000-00011.

26. Jimenez-Almonte JH, King JD, Luo TD, Aneja A, Moghadamian E. Classifications in Brief: Sanders Classification of Intraarticular Fractures of the Calcaneus. Clin Orthop Relat Res. 2019;477(2):467-471. https://doi.org/10.1097/CORR.0000000000000539.

27. Furey A, Stone C, Squire D, Harnett J. Os calcis fractures: analysis of interobserver variability in using Sanders classification. J Foot Ankle Surg. 2003;42(1):21-23. https://doi.org/10.1053/jfas.2003.49999.

28. Bhattacharya R, Vassan UT, Finn P, Port A. Sanders classification of fractures of the os calcis. An analysis of inter- and intra-observer variability. J Bone Joint Surg Br. 2005;87(2):205-208. https://doi.org/10.1302/0301-620x.87b2.15260.

29. Sanders R, Vaupel ZM, Erdogan M, Downes K. Operative treatment of displaced intraarticular calcaneal fractures: long-term (10-20 Years) results in 108 fractures using a prognostic CT classification. J Orthop Trauma. 2014;28(10):551-563. https://doi.org/10.1097/BOT.0000000000000169.

30. Rubino R, Valderrabano V, Sutter PM, Regazzoni P. Prognostic value of four classifications of calcaneal fractures. Foot Ankle Int. 2009;30(3):229-238. https://doi.org/10.3113/FAI.2009.0229.

31. Brunner A, Heeren N, Albrecht F, Hahn M, Ulmar B, Babst R. Effect of three-dimensional computed tomography reconstructions on reliability. Foot Ankle Int. 2012;33(9):727-733. https://doi.org/10.3113/FAI.2012.0727. 


\section{Figures}
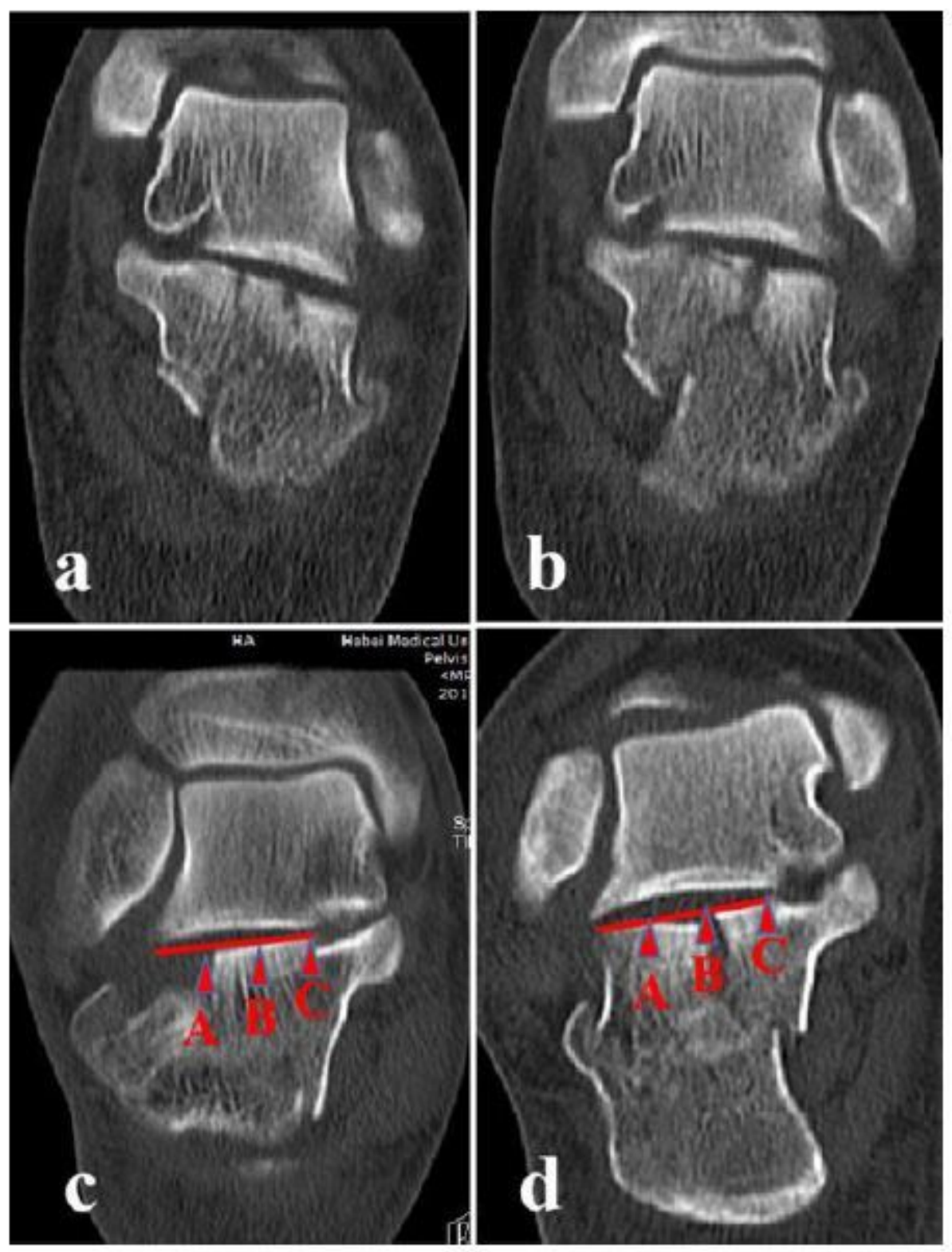

\section{Figure 1}

The coronal sections of the widest part of the posterior facet of the calcaneus (a-d) describe three fracture types that cannot be classified or are controversial in the Sanders FCS. The fracture types identified in two adjacent coronal sections of the CT scanning at the widest part are inconsistent in the same case $(a, b)$. $c$, the fracture line is located around the junction of line $A$, and it is difficult to accurately distinguish between type $\triangle A$ and type $\triangle B$. $d$, the fracture line is located around the junction of line $B$, and it is difficult to accurately distinguish between type $\mathbb{B}$ and type $\triangle \mathrm{C}$. 


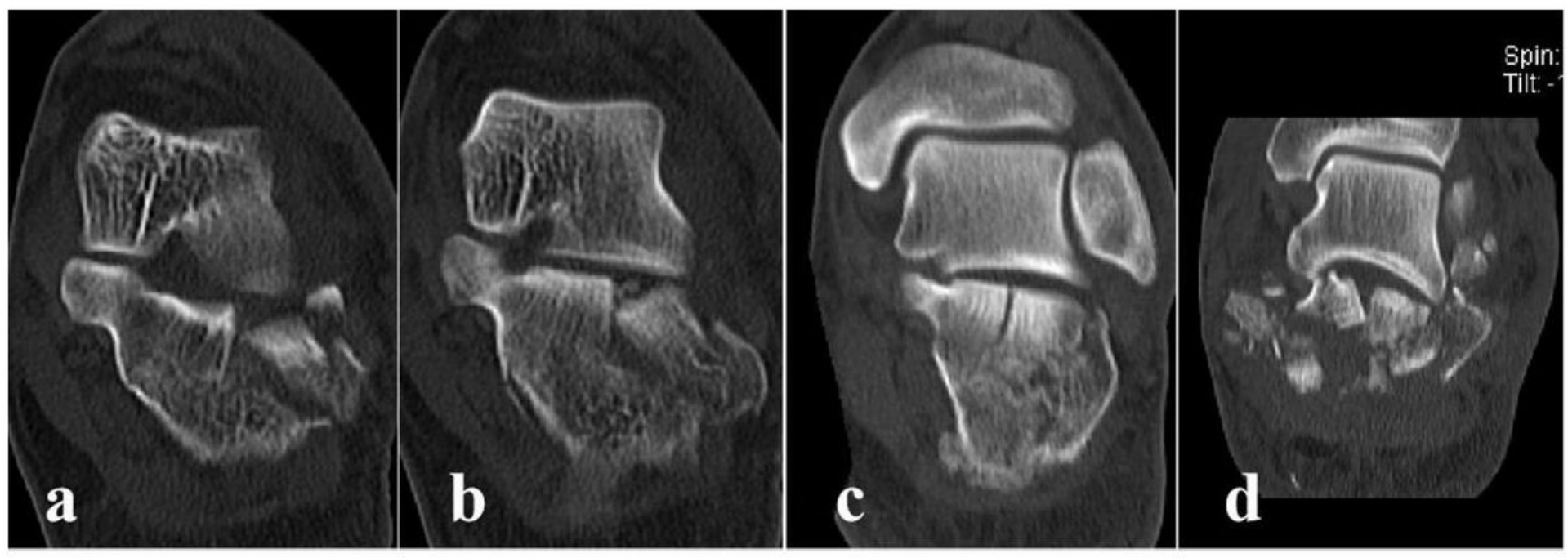

Figure 2

The coronal sections of the posterior facet of the calcaneus (a-d) describe three fracture types that cannot be classified or are controversial in the Eastwood-Atkins FCS. The fracture types identified in two adjacent coronal sections of the CT scan are inconsistent in the same case ( $a$, type $\varangle ; b$, type $\llbracket)$. Nondisplaced (c) and severe comminuted (d) types are not included in the Eastwood-Atkins FCS. 